\title{
Development of Soft X-ray Microanalysis using Windowless SDD Technology
}

\author{
S. Burgess, X. Li, J. Holland, P. Statham, S. Bhadare, D. Birtwistle, A. Protheroe \\ Oxford Instruments NanoAnalysis, Halifax Road, High Wycombe, HP12 3SE, UK
}

New instrumentation which is sensitive to the detection of ultra low energy $(<100 \mathrm{eV}) \mathrm{X}$-rays $[1,2]$, promises new analysis capability for the SEM; so called 'Soft X-ray Microanalysis'. Only those soft X-rays that are generated close to the surface of the sample escape and therefore they offer potential for surface and higher spatial resolution analysis. The detectability of very low energy $\mathrm{K}, \mathrm{L}$ and $\mathrm{M}$ lines also add useful chemical information when operating SEMs at very low accelerating voltage ( $2 \mathrm{kV}$ or less).

To develop EDS hardware that can efficiently detect soft X-rays a number of challenges have been overcome. Although modern SDD detectors are sensitive to low energy X-rays, the absorption of X-rays by the detector window limits the count rate and has a drastic effect for soft X-rays. Therefore, special X-Max ${ }^{N}$ windowless detectors have been fabricated using low noise electronics and high resolution SDD sensors, to maximize ultra low energy sensitivity and deliver more than 10 times improvement at low energy. Performance for SiL $\propto(92 \mathrm{eV})$ and AlL $\propto($ Fig. 1A, 79eV) has been enhanced sufficiently so that these lines may be used for X-ray mapping, and even lower energy lines including LiK $\alpha(54 \mathrm{eV})$ and $\mathrm{MgL} \propto(49 \mathrm{eV})$ have been detected. Recent developments in electronic design have further reduced electronic noise and given additional sensitivity enhancements, as seen for $\operatorname{LiK} \alpha$ (Fig 1B), and $\mathrm{MgL} \propto$ (Fig. 1C).

Soft X-ray Microanalysis presents new challenges, for X-ray emissions involving transitions from valence shells. In particular, the bonding characteristics of the compounds analysed can affect the intensity of X-ray emission. Sample preparation is a vital consideration, with materials of interest often being highly reactive. Analysis of Li- compounds in Li-Al alloys and Li-ion batteries, have yielded results where it is has proved difficult to determine if deficiency of LiK $\alpha$ signal is due to lack of X-ray emission or due to the reactivity of $\mathrm{Li}$ and its loss from the sample surface and we need a better understanding of the physics of soft X-ray emission. For sample preparation, techniques used in surface science, for example the use of in-chamber ion milling, may be required.

Magnesium and aluminium-based alloys are an interesting application area because their metallic bonding means significant soft X-ray L $\propto$ lines are emitted. Dispersoids and other precipitates can also be very small $(<50 \mathrm{~nm})$ in size. Preparation of these materials is more straight-forward using established metallographic techniques; although a thin oxide forms on the surface, useful X-ray intensities are achieved. X-ray maps can be obtained to give element distribution information using very low $\mathrm{kV}$ with the latest FEG-SEMs. Fig. 2A shows the distribution of precipitates in a $\mathrm{Mg}$ alloy from the $\mathrm{MgL} \propto$ line signal collected at $1 \mathrm{kV}$. Smaller nano-scale disperoids from Al-alloys can be better studied using the AlL $\propto$ (rather than the AlK $\alpha$ ) signal at 2$3 \mathrm{kV}$ due to its high intensity. Collection times are limited due to carbon contamination, although preparation methods such as plasma cleaning do reduce the rate of carbon build-up.

Peak shape at low energies is affected by the charge collection mechanism in the detector. SiL $\propto$ and AlL $\propto$ peaks overlap in EDS and work to characterize the low energy response has allowed peak deconvolution for X-ray mapping to be investigated. For example, X-ray maps using windows integrals for AlL $\propto$ (Fig. 3A) and SiL $\propto$ (Fig. 3B), show a false picture of Si- and Cu-rich precipitates in an Alalloy. 
Peak deconvolved 'Tru-Maps' for Al (Fig. 3C) and Si (Fig. 3D), show a more faithful picture of the Xray emission distribution in the sample. However, the $\mathrm{Al}$ emission is influenced by bonding and/or $\mathrm{X}$ ray absorption and the commonly-used tabulations of mass absorption coefficients for microanalysis do not extend below $180 \mathrm{eV}$. Therefore, more investigations are required before we can convert X-ray emission intensity to concentration with confidence at such low energies.

\section{References:}

[1] M. Terauchi et al (2011) Journal of Electron Microscopy, 61 (1), 1-8.

[2] S. Burgess et al (2013) Microscopy and Analysis, 27 (4), S8-S13. (EU).
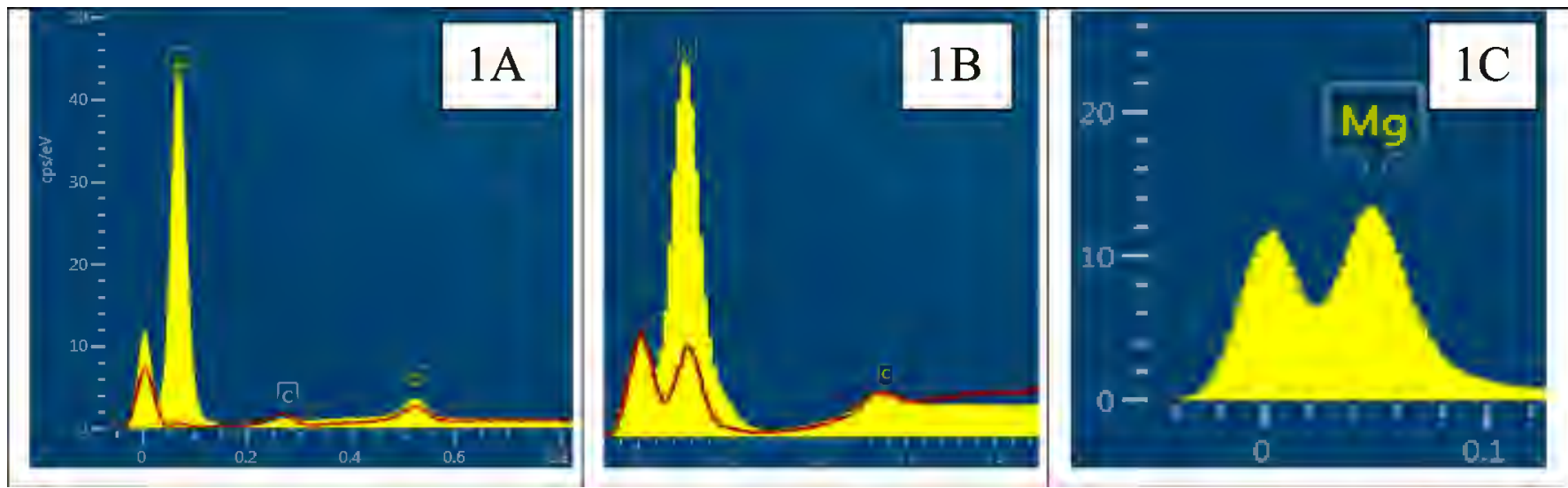

Figure 1. A) AlL $\propto$ intensity comparing windowless (yellow) and thin window SDD (red), B) $\mathrm{LiK} \alpha$ intensity comparing first (red) and second (yellow) generation electronics, C) $\mathrm{MgL} \propto$ emission.

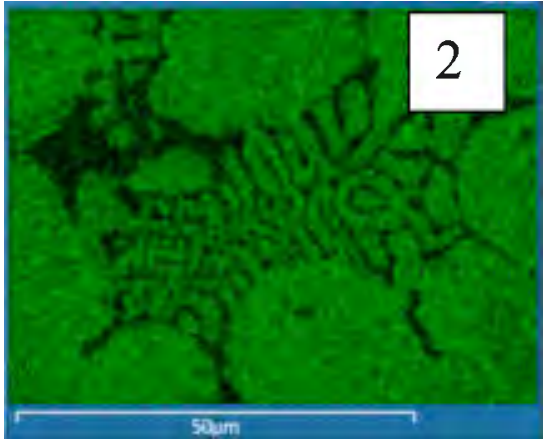

Figure 2. A) MgL $\ell \mathrm{X}$ ray map collected from a $\mathrm{Mg} \mathrm{Zn}$ alloy at $1 \mathrm{kV}$, showing useful X-ray information can be collected when working at very low $\mathrm{kV}$.
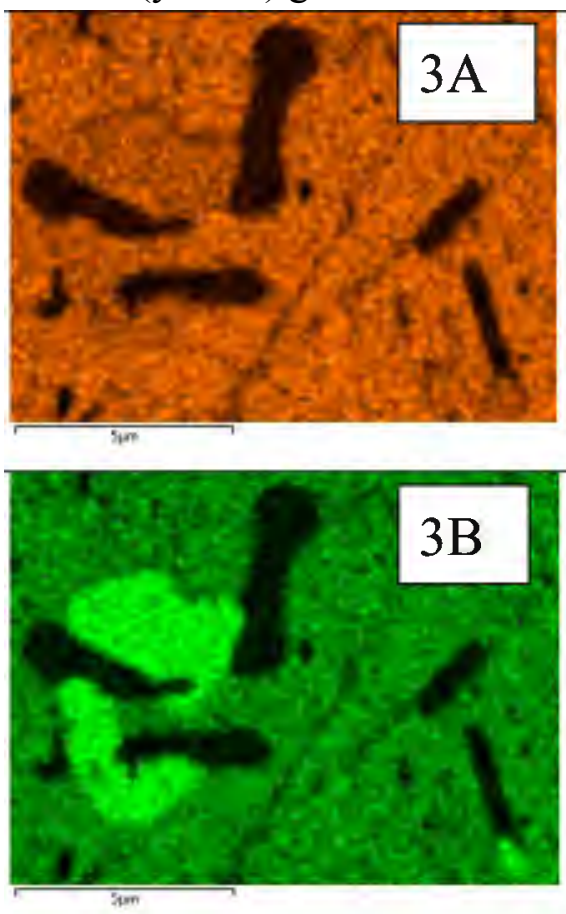
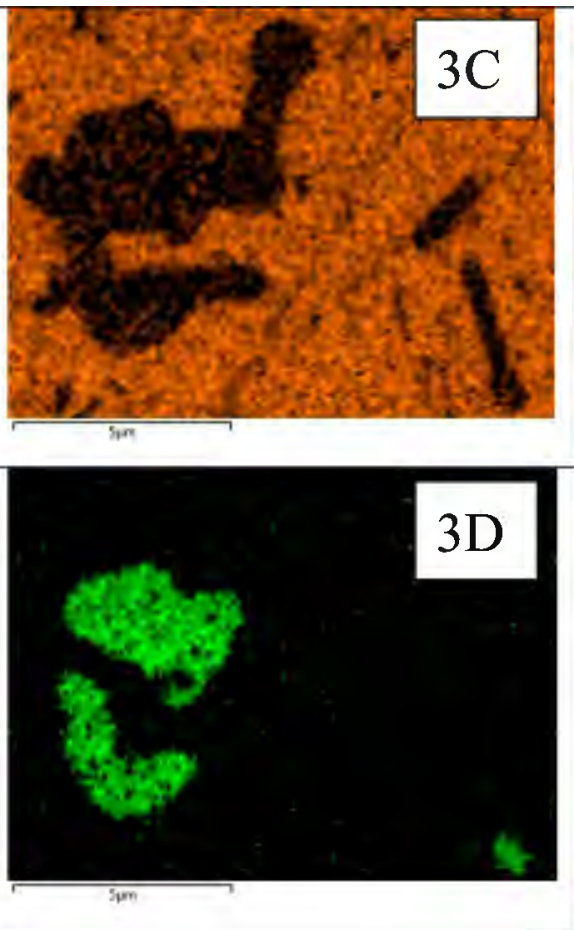

Figure 3. Conventional AlL $\propto$ (A) and $\mathrm{SiL} \propto$ (B) X-ray maps of Si-rich precipitates in an Al-alloy showing incorrect intensity distributions. AlL $\propto(C)$ and $\mathrm{SiL} \propto(\mathrm{D})$ intensity distributions corrected using peak deconvolution at each pixel. 